JHU-TIPAC-96016

hep-ph/9610532

\title{
Quenched Chiral Perturbation Theory for Vector Mesons
}

\author{
Michael Booth, George Chiladze and Adam F. Falk \\ Department of Physics and Astronomy, The Johns Hopkins University \\ 3400 North Charles Street, Baltimore, Maryland 21218 U.S.A.
}

(October 1996)

\begin{abstract}
We develop quenched chiral perturbation theory for vector mesons made of light quarks, in the limit where the vector meson masses are much larger than the pion mass. We use this theory to extract the leading nonanalytic dependence of the vector meson masses on the masses of the light quarks. By comparing with analogous quantities computed in ordinary chiral perturbation theory, we estimate the size of quenching effects, observing that in general they can be quite large. This estimate is relevant to lattice simulations, where the $\rho$ mass is often used to set the lattice spacing.
\end{abstract}




\section{INTRODUCTION}

Lattice QCD has made great progress in recent years, to the point that the computation of the masses of the light hadrons with the accuracy of a few percent is within sight [1]. Of course, such a precision requires that one understand well the various approximations, extrapolations and ansätze which underlie the calculation, in order to estimate, and then minimize, the error which they induce. Without a doubt, the most significant of these, and by far the least well understood, are the corrections due to quenching, particularly as they affect the extrapolation of results to the physical values of the light quark masses.

Quenching errors divide roughly into short and long distance parts. At short distance, their primary effect is to change the running of the coupling constant $\alpha_{s}$. This manifests itself, for instance, in the wave functions of quarkonia, where it can be studied in detail [2]. The long distance effects are by their nature more difficult to quantify. One approach is to use quenched chiral perturbation theory (QChPT), that is, the low energy effective theory of quenched QCD. This idea was proposed by Sharpe [4. 5], with the formalism further developed by Bernard and Golterman [6,7]. The advantage of using a version of chiral perturbation theory to study this problem is that its predictions follow from the basic properties of the underlying theory. Moreover, ordinary chiral perturbation theory is known to describe the low energy dynamics of unquenched QCD very well.

More recently, QChPT has been extended to describe the interactions of pions with baryons [8,9] and heavy mesons [10, [1] in quenched QCD. In this paper, we will extend it further to describe the interactions of the light vector mesons, $\rho, K^{*} \omega$ and $\phi$. Jenkins, Manohar and Wise [12] have formulated ordinary chiral perturbation theory for the vector mesons, treating the vector mesons as heavy particles, in a manner similar to chiral perturbation theory for baryons [13. We will adapt their formalism to quenched QCD. Although we will adhere to most of their conventions, we will use a slightly modified Lagrangian which generalizes more easily to the quenched case and to an arbitrary number $N_{f}$ of light quarks.

What can we expect to learn from such an investigation? The difficulty with low energy effective theories is that typically they contain a large number of undetermined coupling constants, all of which must be fixed from experiment. In fact, only for that sector of chiral perturbation theory which describes the self-interactions of the pions are there enough data to carry out such a phenomenological program beyond leading order. For all other particles and interactions in the chiral Lagrangian, the data are scanty at best. The situation in the quenched theory is even worse, since the only "data" available are extracted from lattice simulations. Hence we are left in a situation where firm and accurate predictions are largely impossible to obtain.

Instead, we take a more modest approach. First, we note that there are certain loop corrections which are predicted unambiguously by the lowest order Lagrangian, namely those that have a nonanalytic dependence on the light quark masses. Such contributions to physical observables cannot be compensated by higher order terms in the chiral expansion. Furthermore, this nonanalytic dependence is particularly sensitive to the long distance behavior of the theory, which is what we wish to investigate. Second, we use the power counting rules which are implicit in chiral perturbation theory, by which all nonperturbative coupling constants are taken to be of order one (given certain other conventions). With

this assumption, we can ask whether the nonanalytic dependence of a physical quantity on 
the light quark masses is small or large. If it is small, we enhance our confidence that the low energy theory is well behaved. However, if it is large, then the applicability of the low energy theory to that quantity is called into question.

The purpose of this investigation, then, is to explore whether QChPT is under reasonable control, by studying those quantities which the theory, at some level, predicts unambiguously. We will find, in fact, that QChPT is quite badly behaved for the masses of the vector mesons, for apparently reasonable values of the nonperturbative coupling constants. Whether this conclusion ought to be extended to quenched QCD itself is not completely clear, but at the very least, our results should sound a serious note of caution about quenched lattice simulations of the vector meson masses.

\section{QUENCHED CHIRAL PERTURBATION THEORY}

The extension of chiral perturbation theory to describe quenched QCD is by now standard. Following Morel [3], for each quark $q^{i}$, a corresponding bosonic "ghost" quark $\tilde{q}^{i}$ is introduced with identical mass, so that the fermion determinant is canceled by the ghost determinant. These ghost quarks will then form mesons with the true quarks and with themselves. As a result, the symmetry group of the theory is enlarged from $S U\left(N_{f}\right)_{L} \times S U\left(N_{f}\right)_{R}$ to the semi-direct product $\left(S U\left(N_{f} \mid N_{f}\right)_{L} \times S U\left(N_{f} \mid N_{f}\right)_{R}\right) \otimes U(1)$.

In this larger theory, the matrix of Goldstone bosons is promoted to a supermatrix,

$$
\Pi=\left(\begin{array}{cc}
\pi & \chi^{\dagger} \\
\chi & \tilde{\pi}
\end{array}\right)
$$

where the quark/ghost content of the fields is $\pi \sim q \bar{q}, \chi^{\dagger} \sim \tilde{q} \bar{q}, \chi \sim q \overline{\tilde{q}}$ and $\tilde{\pi} \sim \tilde{q} \overline{\tilde{q}}$. Each of these is actually an $N_{f} \times N_{f}$ matrix; for example, for $N_{f}=3, \pi$ is the ordinary pseudoscalar nonet

$$
\pi=\left(\begin{array}{ccc}
\frac{1}{\sqrt{2}} \pi^{0}+\frac{1}{\sqrt{6}} \eta & \pi^{+} & K^{+} \\
\pi^{-} & -\frac{1}{\sqrt{2}} \pi^{0}+\frac{1}{\sqrt{6}} \eta & K^{0} \\
K^{-} & \bar{K}^{0} & -\sqrt{\frac{2}{3}} \eta
\end{array}\right)+\frac{1}{\sqrt{3}} \eta^{\prime} I_{3} .
$$

Note that $\chi$ and $\chi^{\dagger}$ are fermionic fields, while $\pi$ and $\tilde{\pi}$ are bosonic.

The Lagrangian of QChPT is given by

$$
\begin{aligned}
\mathcal{L}_{Q \chi}=\frac{f^{2}}{8}( & \left.\operatorname{Str}\left[\partial_{\mu} \Sigma \partial^{\mu} \Sigma^{\dagger}\right]+4 B_{0} \operatorname{Str}\left[\mathcal{M}_{+}\right]\right) \\
& +\frac{1}{2}\left(A_{0} \operatorname{Str}\left[\partial_{\mu} \Pi\right] \operatorname{Str}\left[\partial^{\mu} \Pi\right]-M_{0}^{2} \operatorname{Str}[\Pi] \operatorname{Str}[\Pi]\right) .
\end{aligned}
$$

Here $\Sigma=\xi^{2}, \xi=e^{i \Pi / f}$ (the normalization is such that $f_{\pi} \approx 130 \mathrm{MeV}$ ) while

$$
\begin{aligned}
\mathcal{M} & =\left(\begin{array}{cc}
M & 0 \\
0 & M
\end{array}\right), \\
M & =\operatorname{diag}\left(m_{1}, \ldots, m_{N_{f}}\right),
\end{aligned}
$$

and $\mathcal{M}_{ \pm}=\frac{1}{2}\left(\xi^{\dagger} \mathcal{M} \xi^{\dagger} \pm \xi \mathcal{M} \xi\right)$. The "supertrace" Str is defined with a minus sign for the ghost-antighost fields. The chief difference between the quenched and unquenched theories 
is the presence of the terms involving $\operatorname{Str}[\Pi]=N_{f}^{1 / 2}\left(\eta^{\prime}-\tilde{\eta}^{\prime}\right)$. In the unquenched theory they can be neglected because they describe the dynamics of the $\eta^{\prime}$ meson, which decouples from the theory. But quenching prevents the $\eta^{\prime}$ from becoming heavy and decoupling, so these terms must be retained in QChPT. We have normalized $A_{0}$ and $M_{0}$ so that they have no implicit dependence on $N_{f}$.

The propagators that are derived from this Lagrangian are the ordinary ones, except for the flavor-neutral mesons, where the non-decoupling of $\Pi_{0}$ leads to a curious double-pole structure. It is convenient to adopt a basis for the these mesons corresponding to $q_{i} \bar{q}_{i}$ and $\tilde{q}_{i} \overline{\tilde{q}}_{i}$. Then the propagator in the flavor-neutral sector takes the form

$$
G_{i j}(p)=\frac{\delta_{i j} \epsilon_{i}}{p^{2}-m_{i i}^{2}}+\frac{-A_{0} p^{2}+M_{0}^{2}}{\left(p^{2}-m_{i i}^{2}\right)\left(p^{2}-m_{j j}^{2}\right)},
$$

where $m_{i i}^{2}=2 B_{0} m_{i}$, and $\epsilon_{i}=1$ if $i$ corresponds to a quark and $\epsilon_{i}=-1$ if $i$ corresponds to a ghost. The second term in the propagator is treated as a new vertex, the so-called "hairpin," with the rule that it can be inserted only once on a given line. Note that this term can induce mixing between quark-antiquark and ghost-antighost pairs.

The treatment for vector mesons is similar. They are combined into the supermatrix

$$
N_{\mu}=\left(\begin{array}{cc}
H_{\mu} & K_{\mu} \\
L_{\mu} & \widetilde{H}_{\mu}
\end{array}\right),
$$

with $H_{\mu}$ the usual matrix of vector mesons, which for $N_{f}=3$ is

$$
H_{\mu}=\left(\begin{array}{ccc}
\frac{1}{\sqrt{2}} \rho_{\mu}^{0}+\frac{1}{\sqrt{6}} \phi_{\mu}^{8} & \rho_{\mu}^{+} & K_{\mu}^{*+} \\
\rho_{\mu}^{-} & -\frac{1}{\sqrt{2}} \rho_{\mu}^{0}+\frac{1}{\sqrt{6}} \phi_{\mu}^{8} & K_{\mu}^{* 0} \\
K_{\mu}^{*-} & \bar{K}_{\mu}^{* 0} & -\sqrt{\frac{2}{3}} \phi_{\mu}^{8}
\end{array}\right)+\frac{1}{\sqrt{3}} S_{\mu} I_{3} .
$$

It is convenient to separate the neutral fields $\phi_{\mu}^{8}$ and $S_{\mu}$ into $S U(3)$ octet and singlet pieces. The actual mass eigenstates, for $N_{f}=3$, are mixtures which correspond more closely to the $\omega_{\mu}(\bar{u} u+\bar{d} d)$ and the $\phi_{\mu}(\bar{s} s)$.

We will work in the "heavy vector meson" limit, previously used for ordinary ChPT for vector mesons by Jenkins, Manohar and Wise [12]. In this approximation, the vector mesons are treated as static fields, whose velocity $v^{\mu}$ does not change when absorbing and emitting soft pions. The kinetic and mass terms for the vector meson fields are given by

$$
\begin{aligned}
\mathcal{L}_{\text {kin }}= & -i \operatorname{Str}\left[N_{\mu}^{\dagger}(v \cdot \mathcal{D}) N^{\mu}\right]-i A_{N} \operatorname{Str}\left[N_{\mu}^{\dagger}\right](v \cdot \mathcal{D}) \operatorname{Str}\left[N^{\mu}\right] \\
\mathcal{L}_{\text {mass }}= & \mu_{0} \operatorname{Str}\left[N_{\mu}^{\dagger}\right] \operatorname{Str}\left[N^{\mu}\right]+\mu \operatorname{Str}\left[N_{\mu}^{\dagger} N^{\mu}\right]+\lambda_{1}\left(\operatorname{Str}\left[N_{\mu}^{\dagger}\right] \operatorname{Str}\left[N_{\nu} \mathcal{M}_{+}\right]+\text {h.c. }\right) \\
& +\lambda_{2} \operatorname{Str}\left[\left\{N_{\mu}^{\dagger}, N^{\mu}\right\} \mathcal{M}_{+}\right] .
\end{aligned}
$$

The covariant derivative is

$$
\mathcal{D}_{\mu} N_{\nu}=\partial_{\mu} N_{\nu}+\left[V_{\mu}, N_{\nu}\right]
$$

where

$$
V_{\mu}=\frac{1}{2}\left(\xi \partial_{\mu} \xi^{\dagger}+\xi^{\dagger} \partial_{\mu} \xi\right)
$$


There is also an axial combination,

$$
A_{\mu}=\frac{i}{2}\left(\xi \partial_{\mu} \xi^{\dagger}-\xi^{\dagger} \partial_{\mu} \xi\right)
$$

which transforms homogeneously under the vector subgroup of the flavor symmetry.

The propagator is somewhat different for the heavy vector mesons, but there is still a double pole structure in the flavor-diagonal terms. Let us neglect the $S U(3)$ violating mass terms proportional to $\lambda_{1}$ and $\lambda_{2}$. Then the propagator for the off-diagonal mesons is

$$
\left(v^{\mu} v^{\nu}-g^{\mu \nu}\right) \frac{1}{v \cdot k-\mu},
$$

where $k^{\mu}=P_{N}^{\mu}-\left(M_{N}-\mu\right) v^{\mu}$ is the "residual momentum" of the vector meson $N$. For the flavor-diagonal fields, the propagator is

$$
G_{i j}^{\mu \nu}(k)=\left(v^{\mu} v^{\nu}-g^{\mu \nu}\right)\left[\frac{\delta_{i j} \epsilon_{i}}{v \cdot k-\mu}+\frac{-A_{N} v \cdot k+\mu_{0}}{(v \cdot k-\mu)^{2}}\right] .
$$

The vector mesons have a common "residual mass" $\mu$ at this order. However, we could as easily choose to fix $\mu=0$, absorbing it all into the static phase associated with the heavy vector meson propagator. We will do this from here on.

There are four invariant interactions between the vector mesons and the Goldstone bosons:

$$
\begin{aligned}
\mathcal{L}_{\text {int }}= & i g_{1} \operatorname{Str}\left[N_{\mu}^{\dagger}\right] \operatorname{Str}\left[N_{\nu} A_{\lambda}\right] v_{\sigma} \epsilon^{\mu \nu \lambda \sigma}+\text { h.c. } \\
& +i g_{2} \operatorname{Str}\left[\left\{N_{\mu}^{\dagger}, N_{\nu}\right\} A_{\lambda}\right] v_{\sigma} \epsilon^{\mu \nu \lambda \sigma} \\
& +i g_{3} \operatorname{Str}\left[N_{\mu}^{\dagger}\right] \operatorname{Str}\left[N_{\nu}\right] \operatorname{Str}\left[A_{\lambda}\right] v_{\sigma} \epsilon^{\mu \nu \lambda \sigma} \\
& +i g_{4} \operatorname{Str}\left[N_{\mu}^{\dagger} N_{\nu}\right] \operatorname{Str}\left[A_{\lambda}\right] v_{\sigma} \epsilon^{\mu \nu \lambda \sigma} .
\end{aligned}
$$

Note that we could "unquench" the theory simply by removing the ghost fields and the flavor-singlet $\eta^{\prime}$, which then would receive a large mass from the anomaly. Only the first two interaction terms in $\mathcal{L}_{\text {int }}$ would remain, corresponding to the two interactions considered in Ref. [12].

\section{NONANALYTIC CORRECTIONS TO VECTOR MESON MASSES}

The interactions in the chiral Lagrangian effect the masses of the vector mesons in two ways. First, there are explicit $S U(3)$ violating terms which correspond to local interactions in the theory. One cannot say much about these terms, except to use the available symmetries to constrain them at any given order in the chiral expansion. Second, there are "long distance" corrections which arise from the infrared parts of loop integrals computed using the Lagrangian at leading order. These corrections have a nonanalytic dependence on the light quark masses $m_{i}$, usually of the form $\sqrt{m_{i}}$ or $m_{i} \ln m_{i}$, and thus cannot be canceled by counterterms at any order in the expansion. It is useful to study these corrections, because, while they may not be dominant in general, they at least set a scale for $S U(3)$ violation. As discussed earlier, such terms also correspond to the part of the theory where the effects of 


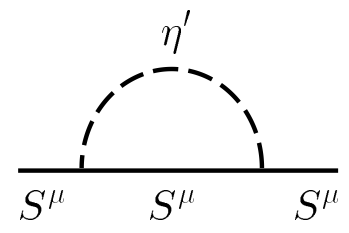

(a)

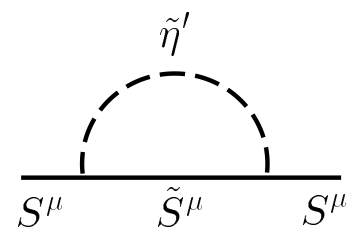

(d)

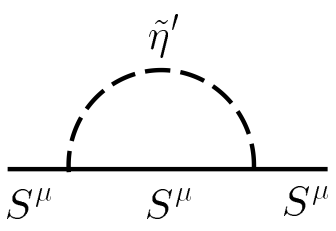

(b)

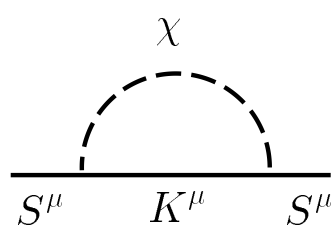

(e)

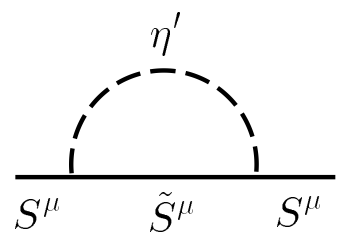

(c)

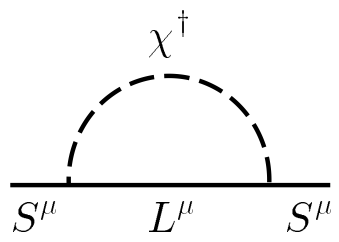

(f)

FIG. 1. Feynman diagrams with no hairpin insertions.

quenching are the least understood. Moreover, they may be dominant for sufficiently small quark masses.

We will use the interaction Lagrangian $\mathcal{L}_{\text {int }}$ to compute the nonanalytic corrections to the vector meson masses for $N_{f}=1,2,3$. We will then compare our result with analogous corrections computed in the unquenched theory. In each case, we will truncate the expansion to include nonanalytic terms of order $m_{q}^{1 / 2}, m_{q} \ln m_{q}$ and $m_{q}^{3 / 2}$, but not those of order $m_{q}^{2} \ln m_{q}$. Where we do not neglect them, we treat the dimensionful parameters $\mu_{0}$ and $M_{0}^{2}$ as being formally of order $m_{q}$.

\section{A. $N_{f}=1$}

The case $N_{f}=1$ is particularly simple, since there are only flavor-singlet mesons. Since it is the flavor-singlet sector which is the source of most complications in QChPT, we work out this case in detail as a pedagogical example.

We begin with the propagator for the Goldstone fields. For the $\eta^{\prime}$ and $\tilde{\eta}^{\prime}$, this is given in matrix form by

$$
\frac{1}{p^{2}-m_{\eta^{\prime}}^{2}}\left(\begin{array}{cc}
1 & 0 \\
0 & -1
\end{array}\right)+\frac{-A_{0} p^{2}+M_{0}^{2}}{\left(p^{2}-m_{\eta^{\prime}}^{2}\right)^{2}}\left(\begin{array}{ll}
1 & 1 \\
1 & 1
\end{array}\right) .
$$

Note the relative minus sign in the non-hairpin parts of the $\eta^{\prime}$ and $\tilde{\eta}^{\prime}$ propagators. The off-diagonal terms mix the $\eta^{\prime}$ and the $\tilde{\eta}^{\prime}$. The propagator for the fermionic $\chi$ field is simply

$$
\frac{1}{p^{2}-m_{\eta^{\prime}}^{2}}
$$

The vector meson propagators are similar. Choosing $\mu=0$, in the bosonic sector we find

$$
\left(v^{\mu} v^{\nu}-g^{\mu \nu}\right) \frac{1}{v \cdot k}\left(\begin{array}{cc}
1 & 0 \\
0 & -1
\end{array}\right)+\left(v^{\mu} v^{\nu}-g^{\mu \nu}\right) \frac{-A_{N} v \cdot k+\mu_{0}}{(v \cdot k)^{2}}\left(\begin{array}{ll}
1 & 1 \\
1 & 1
\end{array}\right) \text {. }
$$

The propagators for the fermions $L_{\mu}$ and $K_{\mu}$ are 


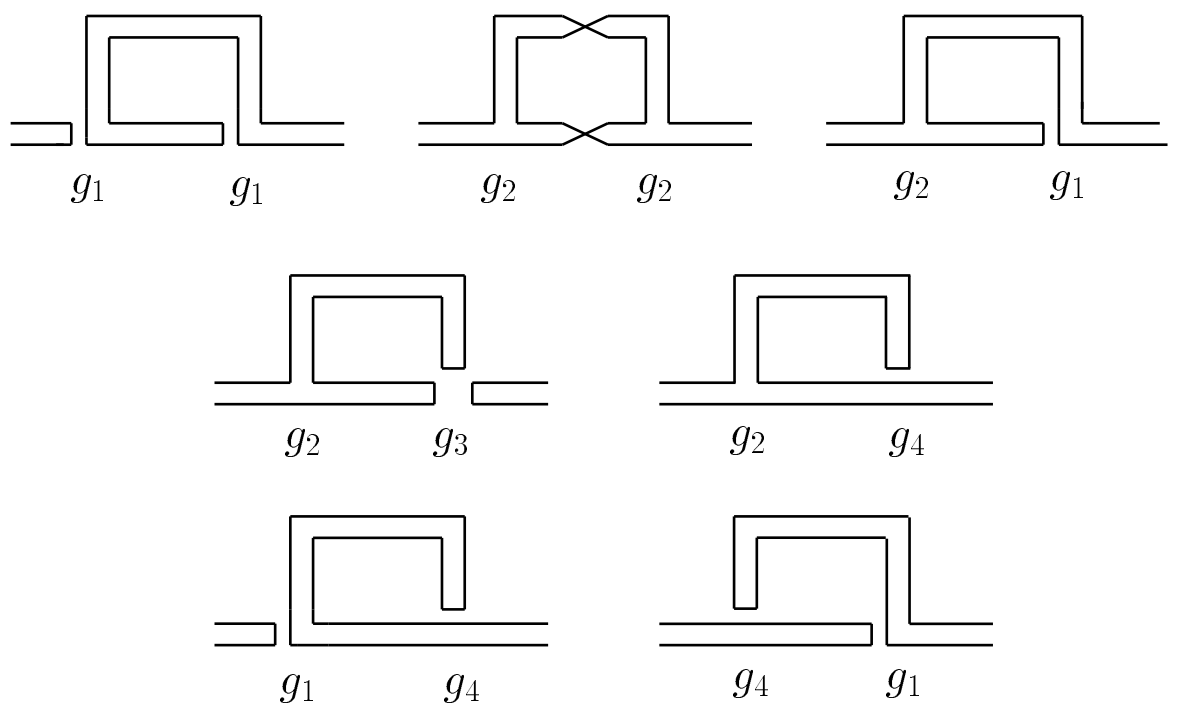

FIG. 2. Quark flow diagrams which contribute to $\delta M_{S}$ in the quenched approximation.

$$
\left(v^{\mu} v^{\nu}-g^{\mu \nu}\right) \frac{1}{v \cdot k} \quad \text { and } \quad-\left(v^{\mu} v^{\nu}-g^{\mu \nu}\right) \frac{1}{v \cdot k},
$$

respectively. A careful study of the ordering of creation and annihilation operators in timeordered products shows that fermion loops containing an $L_{\mu}$ and a $\chi^{\dagger}$ come with the usual minus sign, but those with a $K_{\mu}$ and a $\chi$ do not. In the Feynman diagrams which we will consider, this effectively cancels the extra minus sign in the $K^{\mu}$ propagator.

Next, we expand the interaction Lagrangian $\mathcal{L}_{\text {int }}$. Since we will be computing the corrections to the real vector meson self-energy at one loop, we only keep terms with a least one factor of $S_{\mu}$. Each of the six relevant couplings has a coefficient which is a linear combination of the $g_{i}$ 's. Suppressing the common factor of $v_{\sigma} \epsilon^{\mu \nu \lambda \sigma}$, we find the coefficients

$$
\begin{array}{rll}
S_{\mu}^{\dagger} S_{\nu} \partial_{\lambda} \eta^{\prime} & : & 2 g_{1}+2 g_{2}+g_{3}+g_{4} \\
S_{\mu}^{\dagger} S_{\nu} \partial_{\lambda} \tilde{\eta}^{\prime} & : & -g_{3}-g_{4} \\
S_{\mu}^{\dagger} \tilde{S}_{\nu} \partial_{\lambda} \eta^{\prime} & : & -g_{1}-g_{3} \\
S_{\mu}^{\dagger} \tilde{S}_{\nu} \partial_{\lambda} \tilde{\eta}^{\prime} & : & -g_{1}+g_{3} \\
S_{\mu}^{\dagger} K_{\nu} \partial_{\lambda} \chi+\text { h.c. } & : & g_{1}+g_{2} \\
S_{\mu}^{\dagger} L_{\nu} \partial_{\lambda} \chi^{\dagger}+\text { h.c. } & : & -g_{1}-g_{2} .
\end{array}
$$

There are four types of graphs which contribute to the $S_{\mu}$ self-energy: those with no hairpin insertions, those with a single hairpin insertion (on either the vector meson or the Goldstone boson line), and those with two hairpin insertions (one on each internal line). The six Feynman diagrams with only "ordinary" propagators are shown in Fig. 11. The same loop integral appears in all the graphs,

$$
\delta M_{S} \propto I_{1}\left(m_{\eta^{\prime}}\right)=-\frac{1}{12 \pi f^{2}} m_{\eta^{\prime}}^{3}+\left(\text { analytic in } m_{\eta^{\prime}}^{2}\right) .
$$

The six diagrams contribute to $\delta M_{S}$ with the following coefficients of $I_{1}\left(m_{\eta^{\prime}}\right)$ : 


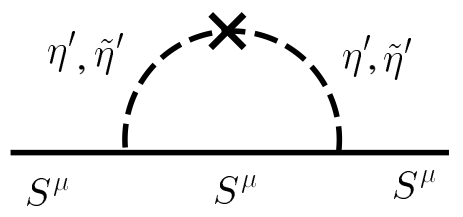

(a)

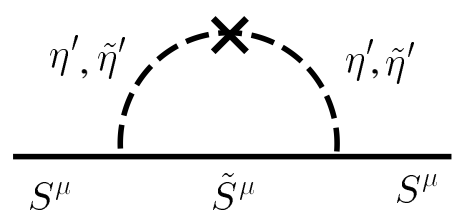

(b)

FIG. 3. Feynman diagrams with a hairpin insertion on the $\eta^{\prime}$ propagator.

$$
\begin{aligned}
& \text { 1(a) : } \quad\left(2 g_{1}+2 g_{2}+g_{3}+g_{4}\right)^{2} \\
& \text { 1(b) : }-\left(-g_{3}-g_{4}\right)^{2} \\
& \text { 1(c) : } \quad-\left(-g_{1}-g_{3}\right)^{2} \\
& \text { 1(d) : }\left(-g_{1}+g_{3}\right)^{2} \\
& \text { 1(e): } \quad-\left(g_{1}+g_{2}\right)^{2} \\
& 1 \text { (f) : } \quad-\left(-g_{1}-g_{2}\right)^{2} \text {. }
\end{aligned}
$$

The minus signs in graphs 1(b) and 1 (c) are from the $\eta^{\prime}$ and $\tilde{S}_{\mu}$ propagators; the minus signs in 1(e) and 1/(f) are from fermion loops. Summing them up, we find

$$
\delta M_{S}=\left(2 g_{1}^{2}+2 g_{2}^{2}+4 g_{1} g_{2}+4 g_{1} g_{4}+4 g_{2} g_{3}+4 g_{2} g_{4}\right) I_{1}\left(m_{\eta^{\prime}}\right)+\ldots
$$

Before we compute the remaining graphs, it is interesting to see which combinations of the $g_{i}$ 's contribute to $\delta M_{S}$. Since the role of the ghost quarks is to implement quenching by canceling the contributions of closed quark loops, the terms which remain after the diagrams have been added should be just those for which no quark loop is necessary. In Fig. 2 we present "quark flow" diagrams corresponding to the combinations of coupling constants which contribute. It is an easy exercise to check that for the combinations which do not appear in $\delta M_{S}$, any quark flow diagram must contain a closed quark loop.

We pause for a moment to consider the dependence of the various terms on the number of colors $N_{c}$. In the large $N_{c}$ limit, the coupling constants scale with $N_{c}$ as follows:

$$
g_{1} \sim \frac{1}{N_{c}}, \quad g_{2} \sim 1, \quad g_{3} \sim \frac{1}{N_{c}^{2}}, \quad g_{4} \sim \frac{1}{N_{c}} .
$$

Hence, it would be tempting to argue that the term proportional to $g_{2}^{2}$ in Eq. (3.8) is dominant in this limit. However, we note from Fig. 2 that the corresponding quark (and color) flow diagram is not planar, so it is also suppressed by $1 / N_{c}$. Of course, this is what we would expect, since in the $N_{c} \rightarrow \infty$ limit the spectrum of the field theory is composed of noninteracting mesons, so Goldstone boson loops should have no effect on the vector meson mass. The lesson is that we must consider more than the coupling constants when extracting the large $N_{c}$ dependence of a term in $\delta M$.

The next class of graphs are those with a hairpin insertion on the $\eta^{\prime}$ line, as shown in Fig. 3. In such a graph, there can be two $\eta^{\prime}$ propagators, two $\tilde{\eta}^{\prime}$ propagators, or (in two ways), an $\eta^{\prime}$ and an $\tilde{\eta}^{\prime}$. So from the graph in Fig. 3 (a), we find a result which is proportional to

$$
\left(2 g_{1}+2 g_{2}+g_{3}+g_{4}\right)^{2}+2\left(2 g_{1}+2 g_{2}+g_{3}+g_{4}\right)\left(-g_{3}-g_{4}\right)+\left(-g_{3}-g_{4}\right)^{2}=\left(2 g_{1}+2 g_{2}\right)^{2},
$$




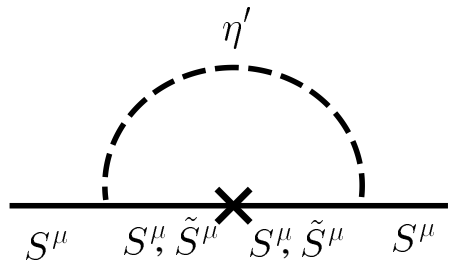

(a)

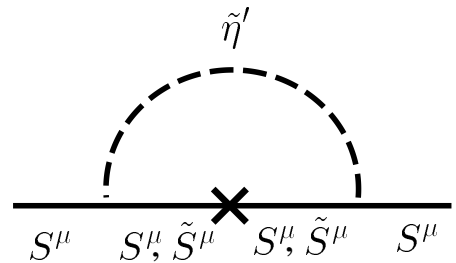

(b)

FIG. 4. Feynman diagrams with a hairpin insertion on the vector meson propagator.

and from Fig. 3(b),

$$
-\left[\left(-g_{1}-g_{3}\right)^{2}+2\left(-g_{1}-g_{3}\right)\left(-g_{1}+g_{3}\right)+\left(-g_{1}+g_{3}\right)^{2}\right]=-\left(-2 g_{1}\right)^{2},
$$

where the minus sign in the second expression comes from the $\tilde{S}_{\mu}$ propagator. Adding these and including the loop integral, we obtain

$$
\delta M_{S}=\ldots+\left(4 g_{2}^{2}+8 g_{1} g_{2}\right) I_{2}\left(m_{\eta^{\prime}}\right)+\ldots,
$$

where

$$
I_{2}\left(m_{\eta^{\prime}}\right)=-\frac{1}{12 \pi f^{2}}\left(\frac{3}{2} M_{0} m_{\eta^{\prime}}-\frac{5}{2} A_{0} m_{\eta^{\prime}}^{3}\right)+\left(\text { analytic in } m_{\eta^{\prime}}^{2}\right)
$$

Similarly, we can have a single hairpin insertion on the vector meson line, as shown in Fig. औ. Here the graph in Fig. G(a) is proportional to

$$
\left(2 g_{1}+2 g_{2}+g_{3}+g_{4}\right)^{2}+2\left(2 g_{1}+2 g_{2}+g_{3}+g_{4}\right)\left(-g_{1}-g_{3}\right)+\left(-g_{1}-g_{3}\right)^{2}=\left(g_{1}+2 g_{2}+g_{4}\right)^{2},
$$

when the $S_{\mu}$ and $\tilde{S}_{\mu}$ propagators are included. The graph in Fig. 4 (b) is proportional to

$$
-\left[\left(-g_{3}-g_{4}\right)^{2}+2\left(-g_{3}-g_{4}\right)\left(-g_{1}+g_{3}\right)+\left(-g_{1}+g_{3}\right)^{2}\right]=-\left(-g_{1}-g_{4}\right)^{2},
$$

where the minus sign is from the $\tilde{\eta}^{\prime}$ propagator. Combining these terms, we find

$$
\delta M_{S}=\ldots+\left(4 g_{2}^{2}+4 g_{1} g_{2}+4 g_{2} g_{4}\right) I_{3}\left(m_{\eta^{\prime}}\right)+\ldots,
$$

where

$$
I_{3}\left(m_{\eta^{\prime}}\right)=\frac{1}{12 \pi f^{2}}\left(\frac{3}{2 \pi} \mu_{0} m_{\eta^{\prime}}^{2} \ln m_{\eta^{\prime}}^{2}+A_{N} m_{\eta^{\prime}}^{3}\right)+\left(\text { analytic in } m_{\eta^{\prime}}^{2}\right) .
$$

Finally, there are graphs with two hairpin insertions, one each on the $\eta^{\prime}$ and $S_{\mu}$ propagators. The sixteen possible graphs are illustrated compactly in Fig. 5. It is straightforward to enumerate them as before, and we find

$$
\delta M_{S}=\ldots+4 g_{2}^{2} I_{4}\left(m_{\eta^{\prime}}\right),
$$

where 


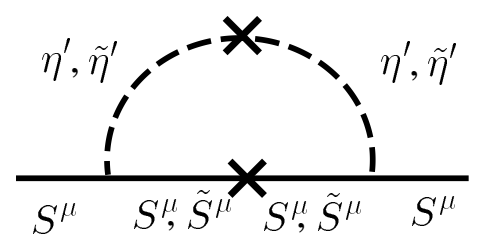

FIG. 5. Feynman diagrams with two hairpin insertions.

$$
\begin{array}{r}
I_{4}\left(m_{\eta^{\prime}}\right)=\frac{1}{12 \pi f^{2}}\left[\frac{3}{2 \pi} \mu_{0} M_{0}^{2}\left(1+\ln m_{\eta^{\prime}}^{2}\right)-\frac{3}{2 \pi} \mu_{0} A_{0} m_{\eta^{\prime}}^{2}\left(1+2 \ln m_{\eta^{\prime}}^{2}\right)\right. \\
\left.+\frac{3}{2} A_{N} M_{0}^{2} m_{\eta^{\prime}}-\frac{5}{2} A_{N} A_{0} m_{\eta^{\prime}}^{3}\right]+\left(\text { analytic in } m_{\eta^{\prime}}^{2}\right) .
\end{array}
$$

In Fig. 6 we present quark flow diagrams corresponding to the surviving diagrams with hairpin insertions, in which there is no closed quark loop. Again, one can convince oneself that all other combinations of coupling constants require quark loops, and hence do not contribute in the quenched theory.

\section{B. $N_{f}>1$}

If there is more than one light flavor, then the situation is complicated by the fact that there is more than one flavor of vector meson. However, we will see the result in this case can be obtained largely by considering the quark flow diagrams in Figs. 2 and 6 for a single flavor.

The matrix of vector mesons $H_{\mu}$ has $N_{f}^{2}$ entries, most conveniently enumerated in the basis $q_{i} \bar{q}_{j}$. In this basis, there are three types of contributions to the mass matrix: $\delta M_{i j}$, $i \neq j$, the correction to the masses of the off-diagonal meson $q_{i} \bar{q}_{j} ; \delta M_{i i}$, the correction to the mass of the flavor-diagonal meson $q_{i} \bar{q}_{j}$; and $\delta M_{i i-j j}, i \neq j$, the correction which mixes the flavor-diagonal mesons $q_{i} \bar{q}_{i}$ and $q_{j} \bar{q}_{j}$. Each of these corrections arises from quark flow diagrams with a particular structure.

The quark flow diagrams in Figs. 2 2 and 6 can be divided into two classes: those in which the quark flavor lines run through from the incoming vector meson to the outgoing one, and those in which they do not. In the first class are the $g_{1} g_{2}$ and $g_{2} g_{4}$ terms in Fig. 2 and the $g_{2}^{2}$ terms in Figs. 6(a) and 6(b). The others are in the second class. Because flavor is conserved in those diagrams in which the quark lines flow through, they contribute to $\delta M_{i j}$ and $\delta M_{i i}$, but not to $\delta M_{i i-j j}$. In the rest of the diagrams, the quark lines in the incoming meson must annihilate in the graph, so they cannot contribute to the off-diagonal $\delta M_{i j}$; however, they do contribute to $\delta M_{i i}$ and $\delta M_{i i-j j}$.

The only other differences in the $N_{f}>1$ case are the somewhat more complicated integrals which arise when a double-pole propagator with $m_{i i} \neq m_{j j}$ appears in a diagram with a hairpin insertion on the Goldstone boson line. The solution is written in terms of integrals with the following nonanalytic dependence on $x^{2}$ and $y^{2}$ :

$$
I_{1}(x)=-\frac{1}{12 \pi f^{2}} x^{3}
$$




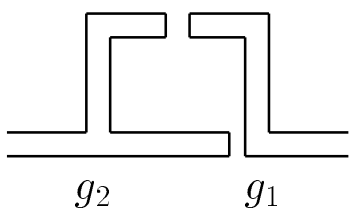

(a)
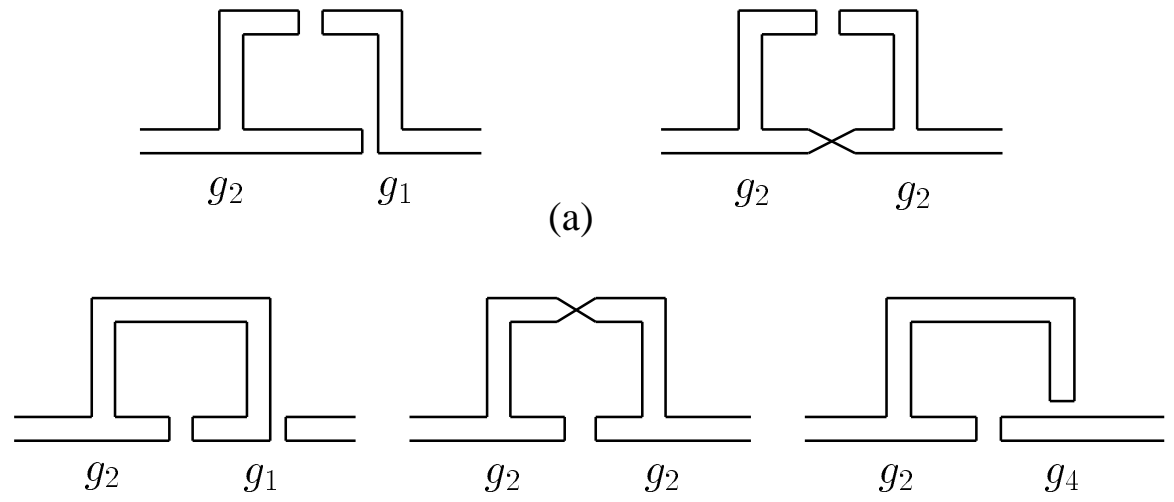

(b)

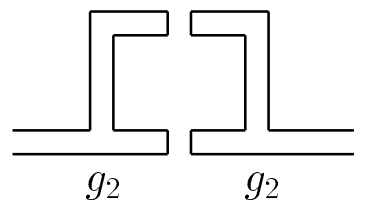

(c)

FIG. 6. Quark flow diagrams corresponding to hairpin insertions. (a) hairpin insertion on the $\eta^{\prime}$ line; (b) hairpin insertion on the $S^{\mu}$ line; (c) two hairpin insertions.

$$
\begin{aligned}
I_{2}(x, y) & =\frac{1}{x^{2}-y^{2}}\left[\left(M_{0}^{2}-A_{0} x^{2}\right) I_{1}(x)-\left(M_{0}^{2}-A_{0} y^{2}\right) I_{1}(y)\right] \\
I_{3}(x) & =\frac{1}{12 \pi f^{2}}\left[\frac{3}{2 \pi} \mu_{0} x^{2} \ln x^{2}+A_{N} x^{3}\right] \\
I_{4}(x, y) & =\frac{1}{x^{2}-y^{2}}\left[\left(M_{0}^{2}-A_{0} x^{2}\right) I_{3}(x)-\left(M_{0}^{2}-A_{0} y^{2}\right) I_{3}(y)\right] .
\end{aligned}
$$

The integrals $I_{2}$ and $I_{4}$ have the degenerate limits

$$
\begin{gathered}
I_{2}(x, x)=-\frac{1}{12 \pi f^{2}}\left[\frac{3}{2} M_{0}^{2} x-\frac{5}{2} A_{0} x^{3}\right] \\
I_{4}(x, x)=\frac{1}{12 \pi f^{2}}\left[\frac{3}{2 \pi} \mu_{0} M_{0}^{2}\left(1+\ln x^{2}\right)-\frac{3}{2 \pi} \mu_{0} A_{0} x^{2}\left(1+2 \ln x^{2}\right)\right. \\
\left.+\frac{3}{2} A_{N} M_{0}^{2} x-\frac{5}{2} A_{N} A_{0} x^{3}\right] .
\end{gathered}
$$

The integrals $I_{i}$ will depend on the variables $m_{i j}=\left[B_{0}\left(m_{i}+m_{j}\right)\right]^{1 / 2}$.

For the masses of the off-diagonal vector mesons, we find the correction

$$
\begin{aligned}
\delta M_{i j}=4 g_{1} g_{2} I_{1}\left(m_{i j}\right)+2 g_{2} g_{4}\left[I_{1}\left(m_{i i}\right)+I_{1}\left(m_{j j}\right)\right] \\
+g_{2}^{2}\left[I_{2}\left(m_{i i}, m_{i i}\right)+2 I_{2}\left(m_{i i}, m_{j j}\right)+I_{2}\left(m_{j j}, m_{j j}\right)\right]+4 g_{2}^{2} I_{3}\left(m_{i j}\right) .
\end{aligned}
$$

The flavor-conserving diagonal mass matrix is perturbed by

$$
\begin{aligned}
\delta M_{i i}=(2 & \left.g_{1}^{2}+2 g_{2}^{2}+4 g_{1} g_{2}+4 g_{1} g_{4}+4 g_{2} g_{3}+4 g_{2} g_{4}\right) I_{1}\left(m_{i i}\right) \\
& +\left(4 g_{2}^{2}+8 g_{1} g_{2}\right) I_{2}\left(m_{i i}, m_{i i}\right)+\left(4 g_{2}^{2}+4 g_{1} g_{2}+4 g_{2} g_{4}\right) I_{3}\left(m_{i i}\right) \\
& +4 g_{2}^{2} I_{4}\left(m_{i i}, m_{i i}\right) .
\end{aligned}
$$


Finally, the contribution to mixing between flavor-diagonal mesons is

$$
\begin{aligned}
\delta M_{i i-j j}=( & \left.g_{1}^{2}+2 g_{2} g_{3}+2 g_{1} g_{4}\right)\left[I_{1}\left(m_{i i}\right)+I_{1}\left(m_{j j}\right)\right]+2 g_{2}^{2} I_{1}\left(m_{i j}\right) \\
& +2 g_{1} g_{2}\left[I_{2}\left(m_{i i}, m_{i i}\right)+2 I_{2}\left(m_{i i}, m_{j j}\right)+I_{2}\left(m_{j j}, m_{j j}\right)\right] \\
& +\left(2 g_{1} g_{2}+2 g_{2} g_{4}\right)\left[I_{3}\left(m_{i i}\right)+I_{3}\left(m_{j j}\right)\right]+4 g_{2}^{2} I_{4}\left(m_{i i}, m_{j j}\right) .
\end{aligned}
$$

\section{Vector meson masses in ordinary chiral perturbation theory}

We would like to compare these results with the nonanalytic corrections to the vector meson masses computed using ordinary chiral perturbation theory. The unquenched case, as discussed in Ref. [12, is somewhat simpler. There are only the fields $H^{\mu}$ and $\pi$, with no $\eta^{\prime}$ field and no hairpin insertions. The interaction Lagrangian is simply

$$
\begin{aligned}
\mathcal{L}_{\chi}= & i g_{1} \operatorname{Tr}\left[H_{\mu}^{\dagger}\right] \operatorname{Tr}\left[H_{\nu} A_{\lambda}\right] v_{\sigma} \epsilon^{\mu \nu \lambda \sigma}+\text { h.c. } \\
& +i g_{2} \operatorname{Tr}\left[\left\{N_{\mu}^{\dagger}, N_{\nu}\right\} A_{\lambda}\right] v_{\sigma} \epsilon^{\mu \nu \lambda \sigma} .
\end{aligned}
$$

Our normalization conventions are chosen to correspond as closely as possible to the quenched Lagrangian, and they differ slightly from those of Ref. [12]. A straightforward calculation yields the nonanalytic corrections to the vector meson mass matrix for $N_{f}=1,2,3$. For $N_{f}=1$, there is only the singlet $S$, and

$$
\delta M_{S}=0 .
$$

For $N_{f}=2$, there are the isotriplet $\rho$ and the singlet $S$. Ignoring isospin violation, we find

$$
\begin{aligned}
\delta M_{\rho} & =2\left(g_{1}+g_{2}\right)^{2} I_{1}\left(m_{\pi}\right) \\
\delta M_{S} & =6\left(g_{1}+g_{2}\right)^{2} I_{1}\left(m_{\pi}\right) \\
\delta M_{\rho-S} & =0 .
\end{aligned}
$$

For $N_{f}=3$, we have

$$
\begin{aligned}
\delta M_{\rho} & =3\left(g_{1}+\frac{2}{3} g_{2}\right)^{2} I_{1}\left(m_{\pi}\right)+g_{2}^{2}\left[\frac{2}{3} I_{1}\left(m_{\pi}\right)+2 I_{1}\left(m_{K}\right)+\frac{2}{3} I_{1}\left(m_{\eta}\right)\right] \\
\delta M_{K^{*}} & =3\left(g_{1}+\frac{2}{3} g_{2}\right)^{2} I_{1}\left(m_{K}\right)+g_{2}^{2}\left[\frac{3}{2} I_{1}\left(m_{\pi}\right)+\frac{5}{3} I_{1}\left(m_{K}\right)+\frac{1}{6} I_{1}\left(m_{\eta}\right)\right] \\
\delta M_{\phi^{8}} & =3\left(g_{1}+\frac{2}{3} g_{2}\right)^{2} I_{1}\left(m_{\eta}\right)+g_{2}^{2}\left[2 I_{1}\left(m_{\pi}\right)+\frac{2}{3} I_{1}\left(m_{K}\right)+\frac{2}{3} I_{1}\left(m_{\eta}\right)\right] \\
\delta M_{S} & =3\left(g_{1}+\frac{2}{3} g_{2}\right)^{2}\left[3 I_{1}\left(m_{\pi}\right)+4 I_{1}\left(m_{K}\right)+I_{1}\left(m_{\eta}\right)\right] \\
\delta M_{\phi^{8}-S} & =\sqrt{3} g_{2}\left(g_{1}+\frac{2}{3} g_{2}\right)\left[3 I_{1}\left(m_{\pi}\right)-2 I_{1}\left(m_{K}\right)-I_{1}\left(m_{\eta}\right)\right] .
\end{aligned}
$$

These expressions for $N_{f}=3$ correspond to those of Ref. [12] if we make the replacement

$$
g_{1} \rightarrow \frac{1}{\sqrt{3}}\left(g_{1}-\frac{2}{\sqrt{3}} g_{2}\right)
$$




\section{PHENOMENOLOGY}

The coefficients which appear in the chiral Lagrangians, both quenched and unquenched, are nonperturbative parameters which are difficult to calculate from first principles. However, we need to have some estimate of their size if we are to use our expressions for phenomenology.

The parameters $A_{0}$ and $M_{0}^{2}$ describing the $\eta^{\prime}$ self-interaction may be studied on the lattice through the hairpin vertex, the anomalous scaling of $m_{\pi}$, and the topological susceptibility $\chi_{t}$ of the pure gauge theory. The effect of the hairpin on $m_{\pi}$ is parameterized by a quantity $\delta$, which is extracted both indirectly and directly. The values of $\delta$ measured on the lattice vary widely [15], in the approximate range $0<\delta<0.3$. With our normalization of the terms in the effective Lagrangian, the relationship between $M_{0}$ and $\delta$ is

$$
M_{0}^{2}=8 \pi f^{2} \delta
$$

from which we find $0<M_{0}<350 \mathrm{MeV}$.

On the other hand, $M_{0}$ is also related to the topological susceptibility $\chi_{t}$ via the WittenVeneziano formula 17,18,

$$
M_{0}^{2}=\frac{4}{f^{2}} \chi_{t}
$$

One must be careful in taking this formula from the original literature, since $\chi_{t}$ is evaluated in the limit $N_{c} \rightarrow \infty$. In this limit $A_{0}=0$, that is, the wavefunction renormalization due to the hairpin vanishes. Hence there is no distinction between writing the left-hand side of Eq. (4.2) as $M_{0}^{2}$ or, instead, as $M_{0}^{2} /\left(1+N_{f} A_{0}\right)$. If we were to take this latter expression, then it would appear that together Eqs. (4.1) and (4.2) determine both $M_{0}$ and $A_{0}$. I However, it is inconsistent to keep the wavefunction renormalization due to $A_{0}$ in one of these relations but not in the other. (If we do so, we are led to the contradictory conclusion that $A_{0} \sim 1 / N_{f}$, while really $A_{0}$ is independent of $N_{f}$.) Instead, Eqs. (4.1) and (4.2) yield two independent determinations of the single variable $M_{0}$. Unfortunately, these determinations are not consistent with each other. Lattice calculations give $\chi_{t} \approx(180 \mathrm{MeV})^{4}$ [16], from which we find $M_{0} \approx 500 \mathrm{MeV}$. As a compromise, we will take $M_{0}=400 \mathrm{MeV}$ in our estimates below.

We have less information on the parameter $A_{0}$. Theoretical prejudice and the large $N_{c}$ limit would lead one to believe that $A_{0}$ is small, but perhaps the inconsistency in the determination of $M_{0}$ is a hint that this limit does not work well here. In his recent review [15], Sharpe fits values for a parameter $\alpha_{\Phi}=A_{0} / 3$ from a number of lattice groups, with the preferred value $A_{0} \sim 0.2$. This is the value we will use in the numerical estimates below, keeping in mind that it is probably highly uncertain.

\footnotetext{
${ }^{1}$ In the notation of Duncan et al. [16], the Lagrangian is written in terms of the parameters $A=1+N_{f} A_{0}$ and $m_{0}^{2}=M_{0}^{2} N_{f} /\left(1+N_{f} A_{0}\right)$. They take $M_{0}^{2} /\left(1+N_{f} A_{0}\right)$ in Eq. (4.2), which corresponds to writing it as $m_{0}^{2}=4 N_{f} \chi_{t} / f^{2}$ instead of as $A m_{0}^{2}=4 N_{f} \chi_{t} / f^{2}$. We agree that Eq. (4.1) corresponds to their expression $A m_{0}^{2}=8 N_{f} \pi^{2} f^{2} \delta$.
} 
We will also assume that $f \approx 130 \mathrm{MeV}$ and $B_{0} \approx 4 \pi f$ are the same in quenched and unquenched chiral perturbation theory, and the same for the coupling constants $g_{1}$ and $g_{2}$. All of the couplings $g_{i}$ are expected to be of order one, with unknown sign. We will assume that there are no dramatic accidental cancellations between different terms, and will take linear combinations of the $g_{i}$ 's to be of order one as well. Finally, we will neglect the vector meson hairpin, setting $A_{N}=0$ and $\mu_{0}=0$. Our primary motivation for this is simplicity; complete expressions in which these terms are included are given above.

\section{A. The quenched $\rho$ mass}

We now use these results to extract information about the effect of quenching in the determination of the $\rho$ mass on the lattice. Quenched chiral perturbation theory gives us information about the nonanalytic dependence of $m_{\rho}$ on the light quark masses. Let us consider a charged $\rho$ in a theory with $N_{f}=2$, the simplest case in which there is no annihilation channel. We will take the two quarks to have equal mass $m_{q}$. Then the expansion of $m_{\rho}$ in terms of $m_{q}$ takes the form

$$
m_{\rho}=\mu+C_{1 / 2} m_{\pi}+C_{1} m_{\pi}^{2}+C_{3 / 2} m_{\pi}^{3}+\ldots,
$$

where the coefficients $C_{i}$ are given by

$$
\begin{aligned}
C_{1 / 2} & =-\frac{M_{0}^{2} g_{2}^{2}}{2 \pi f^{2}} \sim-1.5 \\
C_{1} & =\frac{\lambda_{2}}{B_{0}} \sim 0.1 \times(100 \mathrm{MeV})^{-1} \\
C_{3 / 2} & =-\frac{1}{12 \pi f^{2}}\left[4 g_{2}\left(g_{1}+g_{4}\right)-10 g_{2}^{2} A_{0}\right] \sim-0.1 \times(100 \mathrm{MeV})^{-2},
\end{aligned}
$$

and $m_{\pi}$ is related to $m_{q}$ by $m_{\pi}^{2}=2 B_{0} m_{q}$. For comparison, the leading non-analytic term in the unquenched case is $C_{3 / 2}^{\chi} m_{\pi}^{3}$, with

$$
C_{3 / 2}^{\chi}=-\frac{2\left(g_{1}+g_{2}\right)^{2}}{12 \pi f^{2}} \sim-0.1 \times(100 \mathrm{MeV})^{-2} .
$$

Of course, the numerical estimates are intended only to give a sense of the order of magnitude of the terms. The $C_{1 / 2} m_{\pi}$ term is large, and its sign is predicted by the quenched theory. Moreover, it is the leading term for small quark mass. The other corrections are at the $10 \%$ level. A fit to lattice data would determine the $C_{i}$ 's and check whether they are of the expected size. In Fig. 0 we present the corrections to $m_{\rho}$ as a function of $m_{\pi}$, for a typical set of coupling constants $g_{i}$ of order one. We show results for two different choices of the couplings $M_{0}$ and $A_{0}$. We also show the unquenched result. As Fig. 7 illustrates, it is quite generic to find extremely substantial nonanalytic corrections to $m_{\rho}$ for $m_{\pi} \gtrsim 400 \mathrm{MeV}$. Of course, the possibility of such large quenching corrections in chiral perturbation theory does little to enhance our confidence in the precise extraction of $m_{\rho}$ from lattice simulations of quenched QCD.

\footnotetext{
${ }^{2}$ We note that in the real world, the size of $m_{\rho}-m_{\omega}$ suggests that these terms are indeed small.
} 


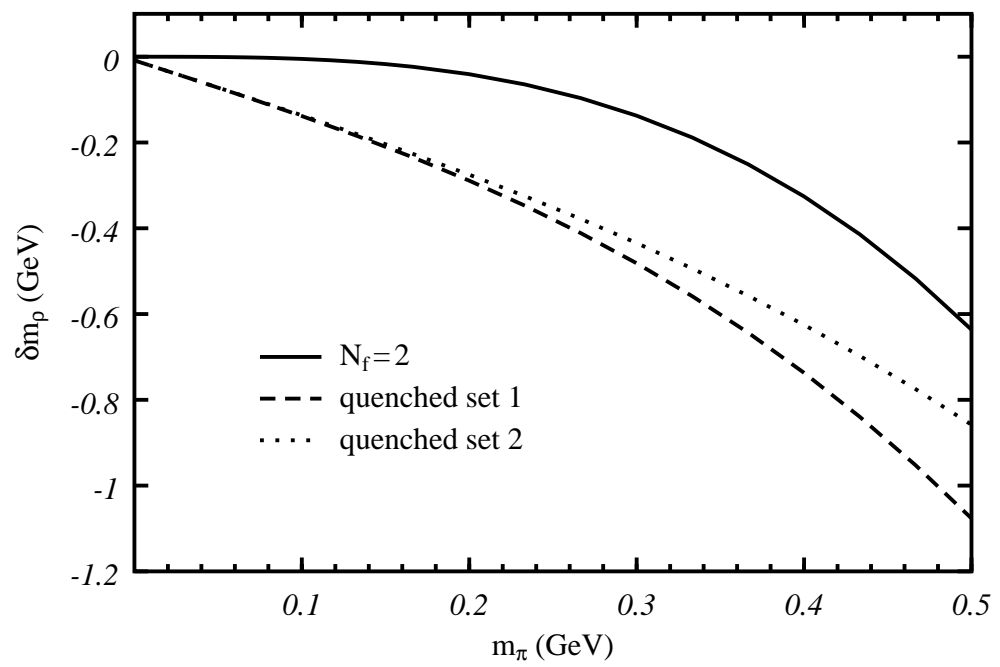

FIG. 7. Correction $\delta m_{\rho}$ to the $\rho$ mass, as a function of $m_{\pi}$, for $N_{f}=2$. The solid line is the result for an unquenched theory; the dashed and dotted lines are for quenched theories with $M_{0}=0.4 \mathrm{GeV}, A_{0}=0$ and $M_{0}=0.1 \mathrm{GeV}, A_{0}=0.2$, respectively. We have taken all $g_{i}=0.75$.

It is also useful to consider the case of unequal quark masses, and to study the quenched chiral corrections to the mass splittings. An interesting quantity to study is $\left(m_{K^{*}}-m_{\rho}\right) /\left(m_{K}^{2}-m_{\pi}^{2}\right)$, which is constant in the chiral limit at leading order. The expression simplifies if we set $m_{\pi}=0$ and study only the dependence on $m_{K}$, in which case we find

$$
\frac{m_{K^{*}}-m_{\rho}}{m_{K}^{2}-m_{\pi}^{2}} \rightarrow \frac{\mathrm{d} m_{K^{*}}}{\mathrm{~d} m_{K}^{2}}=\frac{\lambda_{2}}{B_{0}}-\frac{1}{12 \pi f^{2}}\left[\left(4 g_{1} g_{2}+4 \sqrt{2} g_{2} g_{4}-9 \sqrt{2} g_{2}^{2} A_{0}\right) m_{K}+\frac{7 \sqrt{2}}{2} g_{2}^{2} \frac{M_{0}^{2}}{m_{K}}\right],
$$

where in this expression $m_{K}$ is the one-loop corrected kaon mass. The divergence of the final term as $m_{K} \rightarrow 0$ reflects the unphysical behavior of the quenched theory in the chiral limit.

Finally, we may estimate the size of quenching errors by comparing the nonanalytic corrections to $m_{\rho}$ in the quenched and unquenched theories. Defining the ratio $R_{\rho}=m_{\rho}^{Q} / m_{\rho}^{\chi}$ to be the ratio of the masses in the two theories, we find

$$
\begin{aligned}
R_{\rho} & =1+\mu^{-1}\left[2\left(g_{1}^{2}-g_{2}^{2}+2 g_{2} g_{4}\right) I_{1}\left(m_{\pi}\right)+4 g_{2}^{2} I_{2}\left(m_{\pi}, m_{\pi}\right)\right] \\
& \simeq 1-\frac{1}{6 \pi f^{2} \mu}\left[m_{\pi}^{3}+3 M_{0}^{2} m_{\pi}-5 A_{0} m_{\pi}^{3}\right],
\end{aligned}
$$

for $g_{1}^{2}-g_{2}^{2}+2 g_{2} g_{4}=1$. For the physical meson masses $m_{\pi}=140 \mathrm{MeV}$ and $m_{\rho}=770 \mathrm{MeV}$, this amounts to about a 30\% error from quenching (which, of course, is dominated by the uncertain $M_{0}^{2}$ term). On the other hand, for larger lattice masses such as $m_{\pi} \approx 500 \mathrm{MeV}$ and $m_{\rho} \approx 1 \mathrm{GeV}$, the quenching error is of order $100 \%$. Clearly, our estimate of the error depends on the many undetermined parameters which enter, but it does suggest that one ought to be cautious when using $m_{\rho}$ as a reference quantity to set the scale of the lattice spacing. 


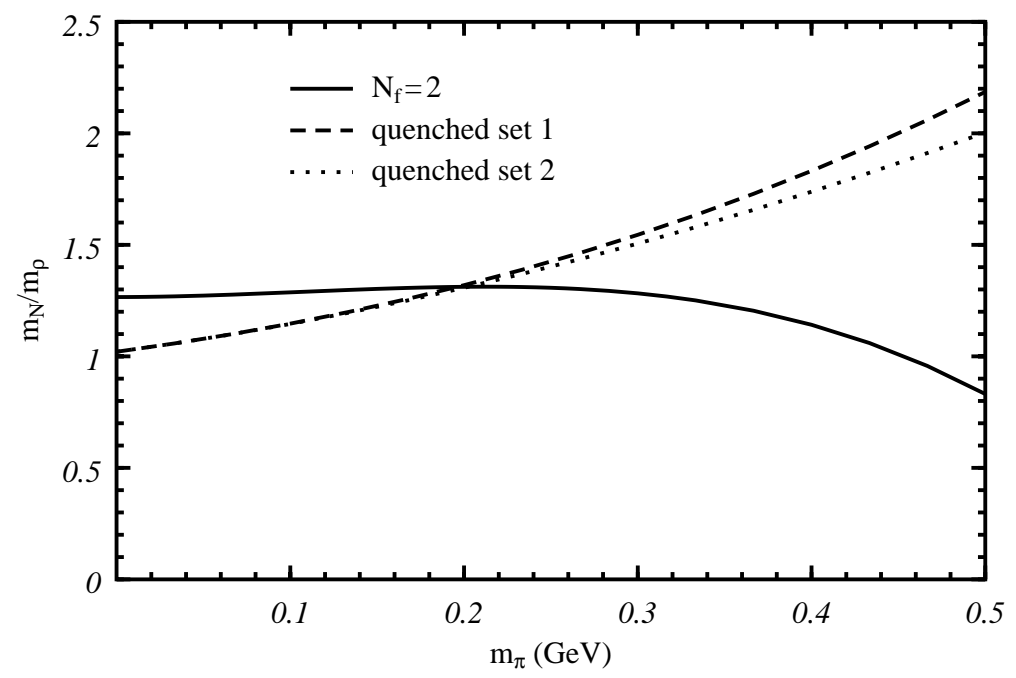

FIG. 8. The ratio $m_{N} / m_{\rho}$ as a function of $m_{\pi}$. The vector meson parameters are as in Fig. 7, while the parameters for the baryon chiral Lagrangian are from Ref. [9].

\section{B. The baryon-meson mass ratio}

The ratio $m_{N} / m_{\rho}$, which experimentally is 1.2 , is used often to gauge the accuracy of lattice simulations. The chiral corrections to $m_{N}$ have been computed by Jenkins [13 and by Bernard, Kaiser and Meissner [14, while the corresponding quenched corrections have been computed by Labrenz and Sharpe [8,9]. Combining these with our calculation, we can examine the quenched chiral corrections to this ratio. We find the expansion

$$
\frac{m_{N}}{m_{\rho}}=\frac{m_{0}}{\mu}+R_{1 / 2} m_{\pi}+R_{1} m_{\pi}^{2}+R_{3 / 2} m_{\pi}^{3}+\ldots
$$

with

$$
\begin{aligned}
R_{1 / 2} & =-\left[\frac{3}{2}(D-3 F)^{2}-4 g_{2}^{2}\right] \frac{M_{0}^{2}}{8 \pi f^{2} \mu} \\
R_{1} & =\left[2\left(b_{D}-3 b_{F}\right)-\frac{\lambda_{2}}{B_{0}}\right] \frac{1}{\mu} \\
R_{3 / 2} & =\left[(D-3 F)(2 D+\gamma)-c^{2}+4 g_{2}\left(g_{1}+g_{4}\right)\right] \frac{1}{12 \pi f^{2}}-\frac{5 A_{0}}{3 M_{0}^{2}} R_{1 / 2},
\end{aligned}
$$

where $D, F, b_{D}, b_{F}, c$ and $\gamma$ are parameters of the baryon chiral Lagrangian [8, 9, 13, 14,. In Fig. 8 we compare the quenched and unquenched corrections, for a typical set of coefficients of order one. Of course, there are far too many unknown parameters for this plot to be anything but illustrative of the possible size of the long distance effects. What the reader

should note is that once again there can easily be a large difference between the two theories, and thus a large correction due to quenching. 


\section{DISCUSSION AND CONCLUSIONS}

Let us close by commenting briefly on our results. The most important qualitative feature is the dependence of $m_{\rho}$ on the square root of the quark mass $m_{q}$. This is to be contrasted with the unquenched theory, where the the leading dependence is linear and the leading nonanalytic dependence goes as $m_{q}^{3 / 2}$. As noted earlier, while the coefficients of these terms depend on nonperturbative parameters, their presence is unambiguously predicted, and they are unchanged by the inclusion of terms of higher order in the chiral expansion. They dominate the extrapolation to small $m_{q}$, and must be accounted for in lattice simulations.

The derivation of our results has relied completely on chiral perturbation theory. The careful reader might worry that because quenched QCD is not a unitary theory, there is no guarantee that QChPT accurately describes its low energy limit. However, there is some independent evidence that QChPT is indeed the low energy theory of quenched QCD. The most celebrated prediction of QChPT, the emergence of "quenched" logarithms of the form $M_{0}^{2} \ln m_{q}$, was originally derived in another way, via strong-coupling perturbation theory [3,4], and the two approaches yield the same coefficient for these terms. We thus expect that the use of QChPT to describe quenched QCD at low energies is valid.

We should also mention one defect of this formulation of chiral perturbation theory for vector mesons, namely the absence of the decay $\rho \rightarrow \pi \pi$. Clearly, this is an important contribution to the width of the $\rho$. However, its contribution to the real part of the $\rho$ mass is rather small. Moreover, a simple one loop calculation shows that the contribution is nonleading, of order $m_{\pi}^{4} \ln m_{\pi}$. We thus expect that the inclusion of this effect would not alter our conclusions substantially.

Since our results depend on many unknown nonperturbative parameters, it is hard to draw firm quantitative lessons from them. Nonetheless, we have found in general that the long distance quenching corrections to vector meson masses could be quite large, at the level of $100 \%$. While it is equally possible that they are small, our analysis does nothing to increase one's confidence that quenching is a controlled error in lattice simulations of $m_{\rho}$ and

$m_{N} / m_{\rho}$. On the contrary, the necessity of simulations of these quantities with dynamical fermions is as pressing as ever.

\section{ACKNOWLEDGMENTS}

This work was supported by the National Science Foundation under Grant No. PHY9404057. A.F. acknowledges additional support from the National Science Foundation for National Young Investigator Award No. PHY-9457916, the Department of Energy for Outstanding Junior Investigator Award No. DE-FG02-94ER40869, and the Alfred P. Sloan Foundation. 


\section{REFERENCES}

[1] S. Gottlieb, Indiana report IUHET-342 (1996), hep-lat/9609306.

[2] A. X. El-Khadra, Nucl. Phys. B (Proc. Suppl.) 26, 372 (1992).

[3] A. Morel, J. Physique 48, 111 (1987).

[4] S. R. Sharpe, Phys. Rev. D 41, 3233 (1990).

[5] S. R. Sharpe, Phys. Rev. D 46, 3146 (1992).

[6] C. W. Bernard and M. F. Golterman, Phys. Rev. D 46, 853 (1992).

[7] C. W. Bernard and M. F. Golterman, Nucl. Phys. B (Proc. Suppl.) 26, 360 (1992).

[8] J. N. Labrenz and S. R. Sharpe, Nucl. Phys. B (Proc. Suppl.) 34, 335 (1993).

[9] J. N. Labrenz and S. R. Sharpe, Phys. Rev. D 54, 4595 (1996).

[10] M. J. Booth, Phys. Rev. D 51, 2338 (1995).

[11] S. R. Sharpe and Y. Zhang, Phys. Rev. D53, 5125 (1996).

[12] E. Jenkins, A. V. Manohar, and M. B. Wise, Phys. Rev. Lett. 75, 2272 (1995).

[13] E. Jenkins, Nucl. Phys. B 368, 190 (1992).

[14] V. Bernard, N. Kaiser, and U. G. Meissner, Z. Phys. C 60, 111 (1993).

[15] S. Sharpe, Washington report UW/PT-96-16 (1996), hep-lat/9609029.

[16] A. Duncan, E. Eichten, S. Perrucci and H. Thacker, hep-lat/9608110 (1996).

[17] E. Witten, Nucl. Phys. B 156, 269 (1979).

[18] G. Veneziano, Nucl. Phys. B 159, 213 (1979); P. Di Vecchia, F. Nicodemi, R. Pettorino and G. Veneziano, Nucl. Phys. B 181, 318 (1981). 